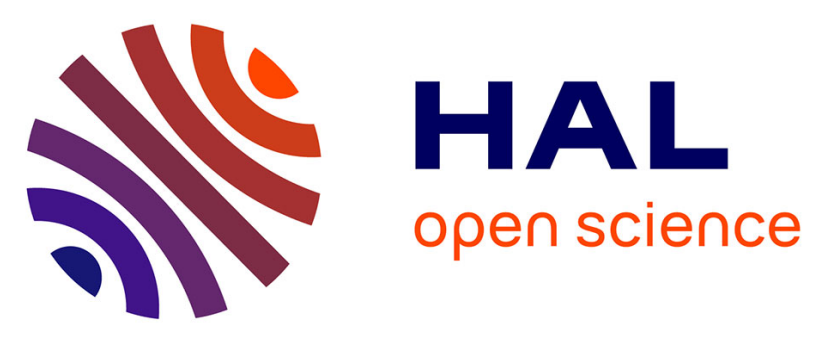

\title{
Spectral micro-scale measurements of the initial stage of streamer-to-filament transition in high pressure nanosecond surface dielectric barrier discharge
}

Chenyang Ding, Antonin Jean, Nicolay Popov, Svetlana Starikovskaia

\section{- To cite this version:}

Chenyang Ding, Antonin Jean, Nicolay Popov, Svetlana Starikovskaia. Spectral micro-scale measurements of the initial stage of streamer-to-filament transition in high pressure nanosecond surface dielectric barrier discharge. AIAA Scitech 2021 Forum, Jan 2021, VIRTUAL EVENT, United States. 10.2514/6.2021-1699 . hal-03437119

\author{
HAL Id: hal-03437119 \\ https://hal.science/hal-03437119
}

Submitted on 19 Nov 2021

HAL is a multi-disciplinary open access archive for the deposit and dissemination of scientific research documents, whether they are published or not. The documents may come from teaching and research institutions in France or abroad, or from public or private research centers.
L'archive ouverte pluridisciplinaire HAL, est destinée au dépôt et à la diffusion de documents scientifiques de niveau recherche, publiés ou non, émanant des établissements d'enseignement et de recherche français ou étrangers, des laboratoires publics ou privés. 


\title{
Spectral micro-scale measurements of the initial stage of streamer-to-filament transition in high pressure nanosecond surface dielectric barrier discharge
}

\author{
Chenyang Ding*, Antonin Jean ${ }^{\dagger}$ \\ Laboratory of Plasma Physics (CNRS, Ecole Polytechnique, Univ. Paris-Sud, Observatoire de Paris, Sorbonne \\ Université, l'Institut Polytechnque de Paris), Ecole Polytechnique, route de Saclay, 91128 Palaiseau, France
}

Nicolay Popov

Skobeltsyn Institute of Nuclear Physics, Moscow State University, Moscow, 119991, Russia. Joint Institute for High

Temperatures of the Russian Academy of Sciences, Moscow, 125412, Russia

Svetlana Starikovskaia ${ }^{\S}$

Laboratory of Plasma Physics (CNRS, Ecole Polytechnique, Univ. Paris-Sud, Observatoire de Paris, Sorbonne

Université, l'Institut Polytechnque de Paris), Ecole Polytechnique, route de Saclay, 91128 Palaiseau, France

The paper presents the recent study of spectral composition of the filament during its propagation process in the single shot surface nanosecond dielectric barrier discharge (nSDBD) at high pressures. The micro-images and spectra at specific moments were recorded by the ICCD camera and spectrometer accompanied with the long distance microscope. This is the first time that we analysis nSDBD in the micrometer scale. The discharge starts with the streamer generated from the high-voltage electrode. A few nanoseconds later, there are a few protrusions passing through the streamer front. After the protrusions' propagation, the filaments are formed in the whole channel connecting the high-voltage electrode and the tip of protrusions. It is found that in the early stage of the streamer-to-filament transition, both the streamer and the protrusion are contributed by the second positive system of nitrogen while only when the whole filament is formed, the cw emission can be seen.

\section{Introduction}

$\mathrm{N}$ ANOSECOND surface dielectric barrier discharge (nSDBD) at high pressures was found to be a possible solution for plasma-assisted ignition and ignition of combustible mixtures in [1-5]. At elevated pressures and voltages, the discharge channel start to constrict and a regular structure of tens of filaments appear from the edge of the high-voltage (HV) electrode and propagate in the direction perpendicular to it. The number of filaments is 4-5 times less than the number of streamers. The so-called streamer-to-filament transition is a general feature of nanosecond discharges for both negative and positive polarities at elevated pressures [6]. The transition is observed in a single-shot experiment a few nanoseconds after the start of the discharge. During the transition process, typically 1-3 ns depending on experimental conditions, the emission spectra changes from molecular bands (e.g. second positive system of nitrogen) to the continuous wavelength $(\mathrm{cw})$ emission with well distinguished broadened lines of atoms $(\mathrm{N}, \mathrm{O}, \mathrm{H})$ and singly ionized atoms, mainly $\mathrm{N}^{+}$. It was shown that the continuous wavelength emission comes from the narrow near-axis zone of the filament. A recent calculation shows that stepwise ionization and dissociation from electronically excited states of molecular nitrogen leads to fast increase of the electron density, dissociation degree and gas heating that is corresponding to the spectral changing [7].

The aim of the present paper is to study the changing of spectral composition of discharge channels from the micrometer scale and to explain the transforming process of streamer-to-filament transition.

\footnotetext{
*Graduate student, Laboratory of Plasma Physics, CNRS, Ecole Polytechnique.

${ }^{\dagger}$ Undergraduate student, Laboratory of Plasma Physics, CNRS, Ecole Polytechnique.

${ }^{\ddagger}$ Senior researcher, Skobeltsyn Institute of Nuclear Physics, Moscow State University.

${ }^{\S}$ Senior researcher, CNRS, Ecole Polytechnique.
} 


\section{Experimental Setup}

This section provides the description of different experimental components used during the experiments. Figure 1 (a) presents the scheme of the electrode system. The metal gear, $20 \mathrm{~mm}$ in diameter, served as the high-voltage electrode in the experiments. Instead of the disc electrode used in [6, 8], the gear-shape HV electrode was used to fix the start position of the discharge which is necessary for spectroscopy experiments. The low-voltage (LV) ground electrode has $20 \mathrm{~mm}$ internal diameter and $46 \mathrm{~mm}$ external diameter. The dielectric PVC film $(\varepsilon=3-3.5, d=0.3 \mathrm{~mm}$ in thickness), was glued to the ground electrode by Geocel FIXER Mate silicon glue with the similar permittivity. The electrode system was installed in a constant volume high-pressure chamber (pressure range from $100 \mathrm{mbar}$ to $12 \mathrm{bar}$ ). The chamber was equipped with three quartz windows which allows optical measurement experiments. The high-voltage pulse with $20 \mathrm{~ns}$ in duration, $2 \mathrm{~ns}$ rising time, was generated from the generator FPG20-03NM for negative polarity or FPG20-03PM for positive polarity (FID technology) and transformed to the electrode via a $30 \mathrm{~m}$ long $50 \Omega$ coaxial cable. The spectral results were recorded by ACTON 2500i spectrograph accompanied with the Pi-Max4 ICCD camera (Princeton Instruments).

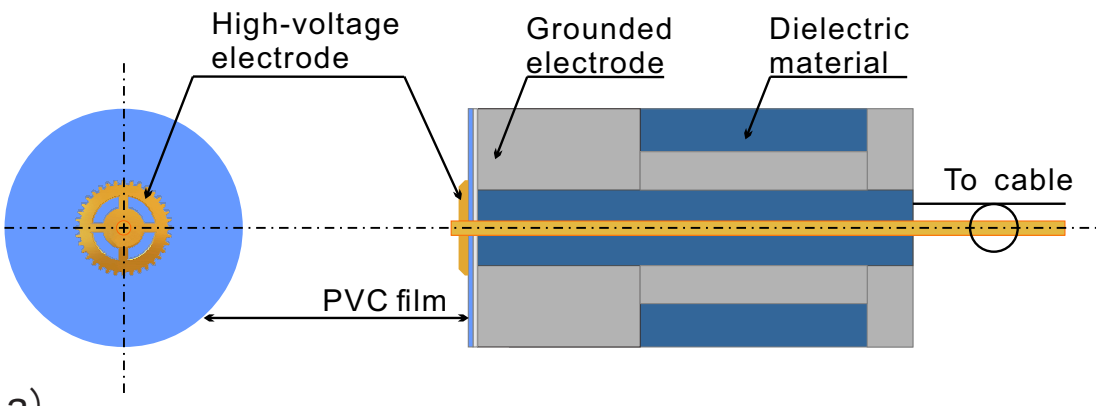

a)

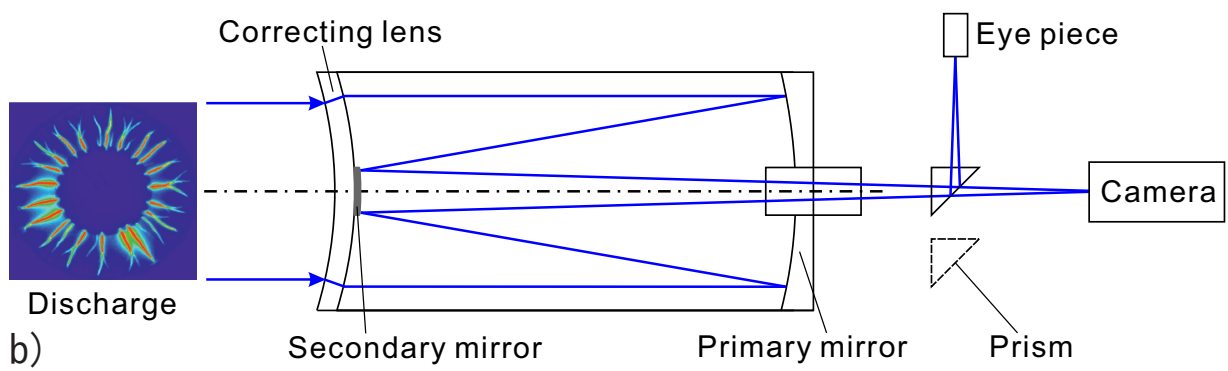

Fig. 1 (a) Schematic of the electrode system; (b) Working principle of the LDM system.

The QM 1 long distance microscope (LDM) (Questar Corporation) accompanied with a few Barrlow lenses and band pass filters was used to study the discharge at the micro scale. The working principle of QM 1 long distance microscope is shown in figure 1 (b). The light enters the optical system via the correcting lens in head of the microscope. After being refracted to the system, the light is reflected by the primary mirror locating at the rear of the microscope. This light is then sent back to the tube in the center of the primary mirror by the reflection of secondary mirror. This configuration gives the possibility of creating a long optical path in a mechanically short system, allowing the increase of the magnification. The prism is used for rotating the light path from horizontal position to vertical position. Instead of entering to the camera, the light would go directly to the eye piece (Questar, $16 \mathrm{~mm}$ focal length, 1.25 in in diameter) with the prism. To analysis the spectral composition of the discharge, a set of UV/VIS bandpass filters (Thorlabs, FB340 - 10, FB380 - 10, FB390 - 10, FL514.5 - 10 and FB750 - 10) were used in the experiments. The filter was placed between the Barlow lenses and ICCD camera.

\section{Results and Discussion}

To analyze the spectral composition of the filamentary discharge, a few band pass filters (Thorlabs) were used. The band pass filter, $10 \pm 2 \mathrm{~nm}$ of FWHM, with the central wavelength of $340 \pm 2 \mathrm{~nm}$ and $390 \pm 2 \mathrm{~nm}$ were used to detect the second positive system and first negative system of nitrogen respectively while the band pass filter with the central wavelength of $514.5 \pm 2 \mathrm{~nm}$ and $750 \pm 2 \mathrm{~nm}$ were used to detect $\mathrm{cw}$ emission. 


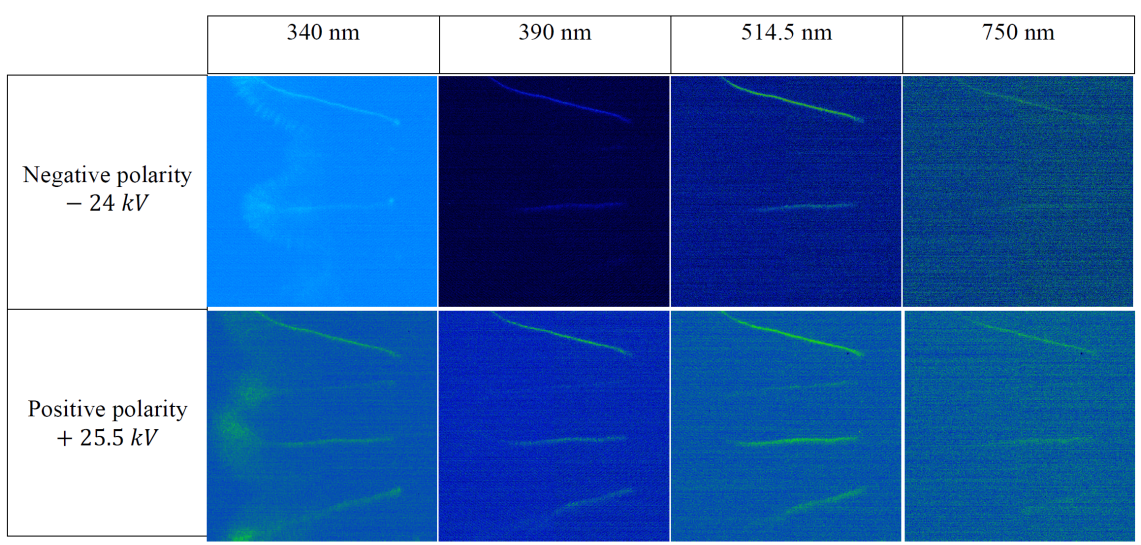

Fig. 2 Micro-scale ICCD images at $t=5 \mathrm{~ns}$ taken by QM 1 LDM with filters, in $\mathbf{N}_{2}, P=6$ bar. Resolution of optical system is $7.6 \mu \mathrm{m} / \mathrm{px}$.

As shown in the first column of figure 2 the streamer phrase (second positive system of nitrogen) can be clearly seen at $U= \pm 25 \mathrm{kV}$. The difference between two polarities is the shape of the streamer front. The feather like structure can be seen better at negative polarity, which was reported in [9]. And for rest columns of figure 2, discharge only appears at the central channels rather than the propagation front. It can be concluded from figure 2 that in the early stage of filament generation, the main discharge channel is the composition of molecule bands and cw emission while the streamer clouds in front of the discharge propagation only has the nitrogen molecule bands.

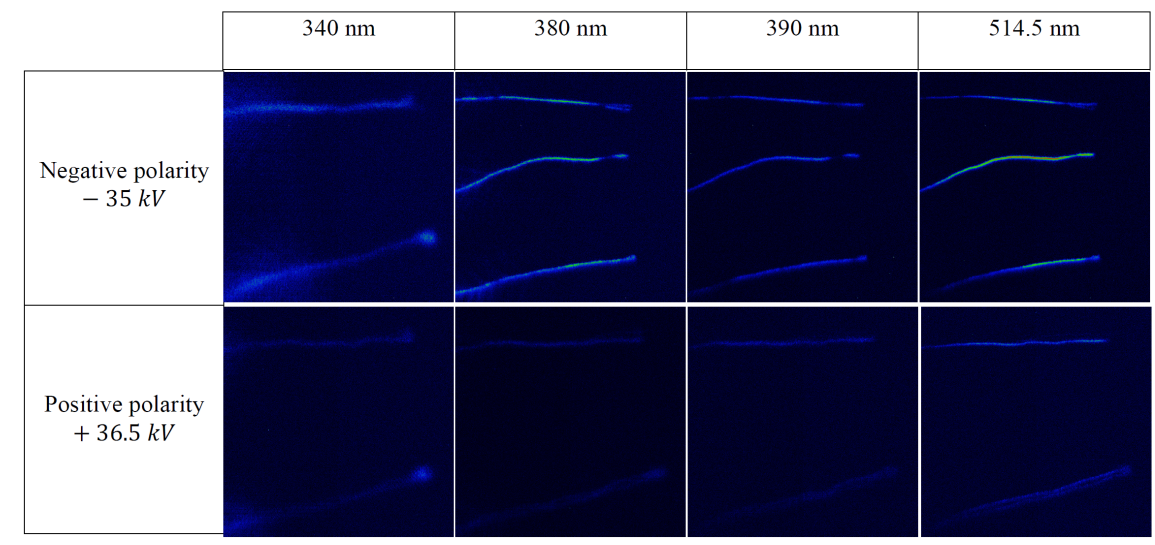

Fig. 3 Micro-scale ICCD images at $t=7 \mathrm{~ns}$ taken by QM 1 LDM with filters, in $\mathbf{N}_{2}, P=6$ bar. Resolution of optical system is $7.6 \mu \mathrm{m} / \mathrm{px}$.

Figure 3 presents the micro-scale ICCD images at $t=7 \mathrm{~ns}$ taken by long distance microscope. The experiments were conducted in 6 bar nitrogen at negative polarity $(35 \mathrm{kV})$ and positive polarity $(36.5 \mathrm{kV})$ with band pass filters $(340 \pm 2 \mathrm{~nm}, 380 \pm 2 \mathrm{~nm}, 390 \pm 2 \mathrm{~nm}$ and $514.5 \pm 2 \mathrm{~nm})$. At higher amplitude of voltages, the streamer front was replaced by a few well-defined filamentary channels. Comparing the results from first column (filter $340 \pm 2 \mathrm{~nm}$ ) and others, we can find that the streamer channels $\left(2^{+} \mathrm{N}_{2}\right)$ are much wider than the filament channels (cw emission) but the emission intensity of streamer is much lower. When comparing the images between different polarities, one can see that the $\mathrm{cw}$ emission of filaments at negative polarity is much obvious than at positive polarity.

In order to analyse the spectral composition of discharge propagation in detail, the discharge at two specific moments ( $t=7 \mathrm{~ns}$ and $11 \mathrm{~ns}$ ) were recorded. The micro-images as well as the spectra in the range of $300-600 \mathrm{~nm}$ were displayed in figure 4 and 5 For the discharge at $t=7 \mathrm{~ns}$, the channel has two zones (I and II) with different structures. Zone I is a bright spot near the HV electrode while zone II forms a arrowhead structure with the protrusion just passing through the streamer front. When comparing the spectra of these two zones, one can see that these two spectra have nearly the same composition of second positive system of nitrogen even though the spectra from zone I is noisier than zone II due to the 

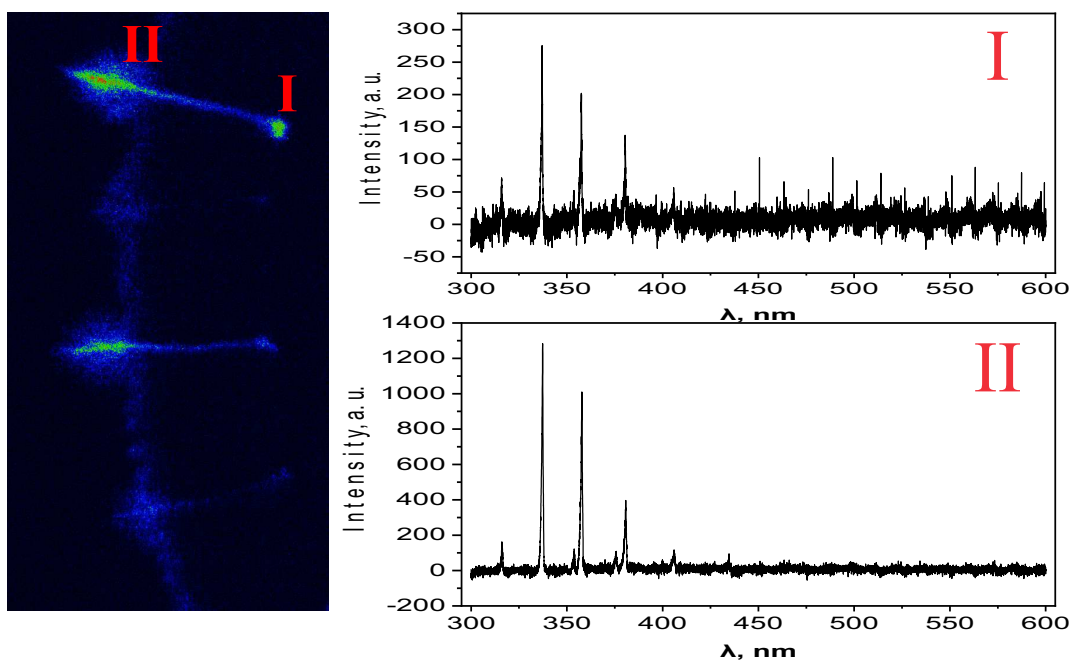

Fig. 4 ICCD image and spectra of one discharge channel at $t=7 \mathrm{~ns}$ for $U=-25 \mathrm{kV}, 6$ bar nitrogen.
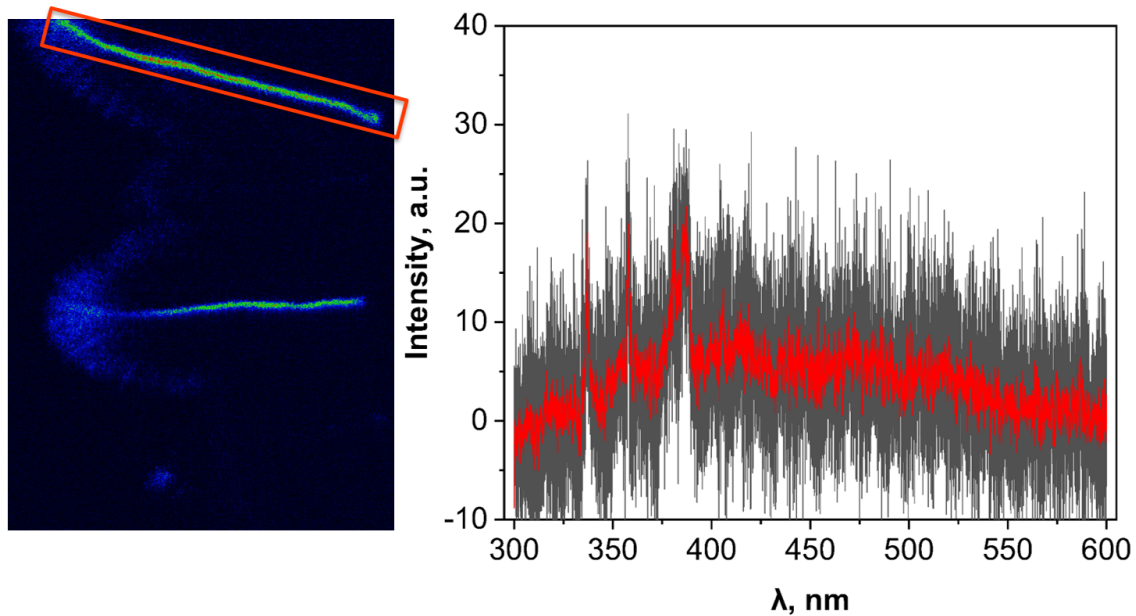

Fig. 5 ICCD image and spectra of one discharge channel at $t=11 \mathrm{~ns}$ for $U=-25 \mathbf{k V}, 6$ bar nitrogen.

less emission intensity.

For the discharge at $t=11 \mathrm{~ns}$, the filament has been well-formed and the emission intensity is distributed homogeneously along the channel. After integrating the whole channel, the spectra can be seen from figure 5 . Different from the spectra of discharge at $t=7 \mathrm{~ns}$, the spectra of discharge $t=11 \mathrm{~ns}$ contains both $\mathrm{cw}$ emission and second positive system of nitrogen. This gives us the idea that a few nanoseconds after the protrusions, the stepwise ionization and dissociation process happen when the filamentary channel is formed. At this time, the ionized nitrogen atoms $\left(\mathrm{N}^{+}\right)$ were produced.

It has already been seen from figure 3 and talked above that the discharge channels would shrink during the streamer-to-filament transition process. It can be seen from figure 6, that the diameter of the discharge channel would also vary depending on the amplitude of applied voltages and pressures. Figure 6(a)(b)(c) show the full width at half maximum (FWHM) of discharge channels at negative $25 \mathrm{kV}, 6$ bar nitrogen (black curve) and negative $35 \mathrm{kV}, 7$ bar nitrogen (red curve) at different positions ( $300 \mu \mathrm{m}, 500 \mu \mathrm{m}$ and $1 \mathrm{~mm}$ to the high-voltage electrode). The images were processed by an open-source software called 'Fiji' which is intended for the imaging analysis [10]. Figure 6(d) is the micro-image taken from the experiment at negative $35 \mathrm{kV}, 7$ bar nitrogen at $t=2.7 \mathrm{~ns}$. The yellow line marks the position of data retrieve, $500 \mu \mathrm{m}$. Figure 6(e) shows the intensity distribution along the yellow line in figure 6(d).

At $t=1 \mathrm{~ns}$, the streamer has just propagated to $300 \mu \mathrm{m}$ to the HV electrode. As the time went from $t=1 \mathrm{~ns}$ to 

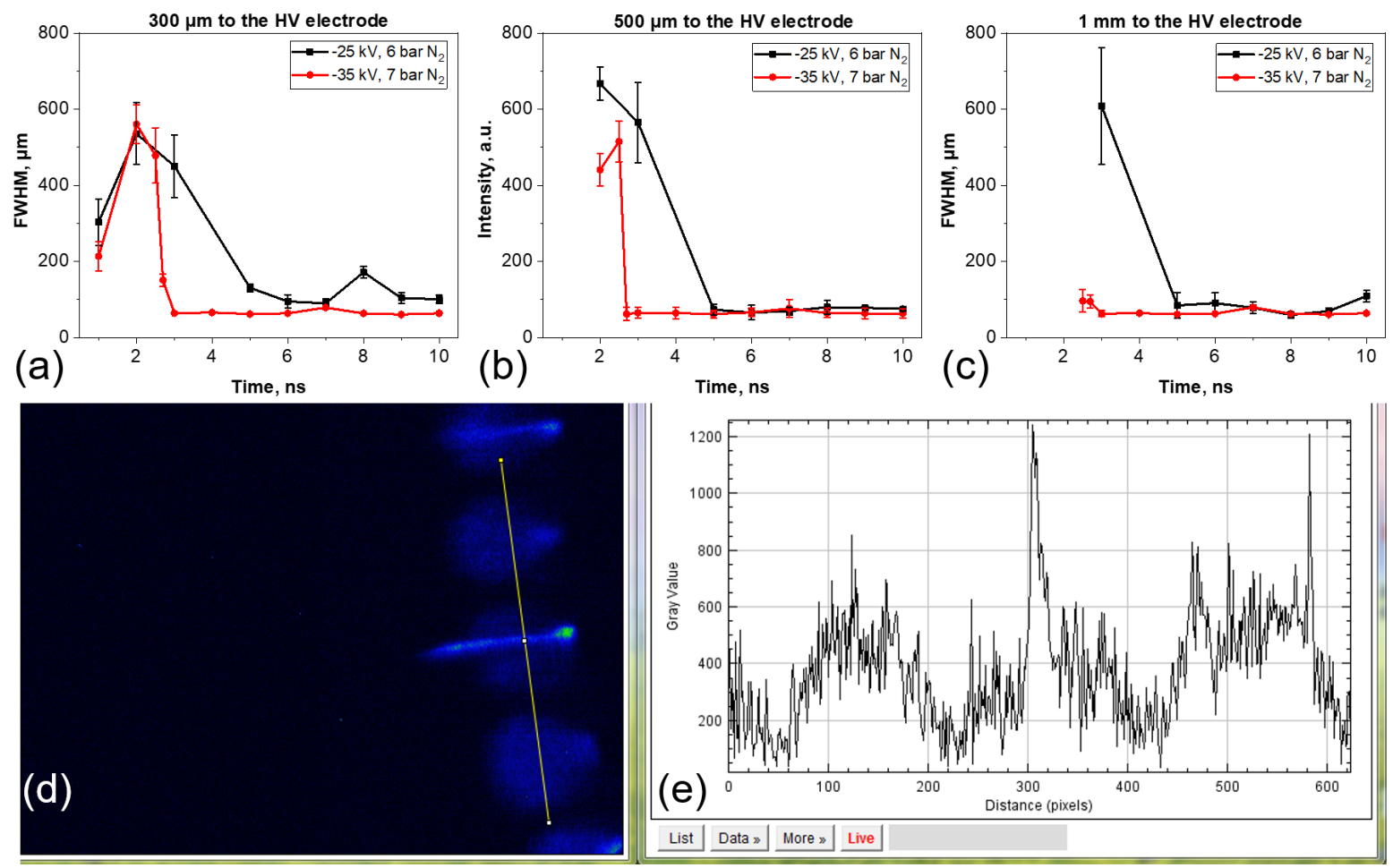

Fig. 6 Full width at half maximum (FWHM) of discharge channels at negative $25 \mathrm{kV}, 6$ bar nitrogen (black curve) and negative $35 \mathrm{kV}, 7$ bar nitrogen (red curve) at different positions (a) $300 \mu \mathrm{m}$; (b) $500 \mu \mathrm{m}$ and (c) $1 \mathrm{~mm}$ to the high-voltage electrode. (d) Micro-image taken from the experiment at negative $35 \mathrm{kV}, 7 \mathrm{bar}$ nitrogen at $t=2.7$ ns. (e) Emission intensity distribution along the yellow line in (d).

$t=2.5 \mathrm{~ns}$, the discharge channel at this position expanded from $200-300 \mu \mathrm{m}$ up to $550 \mu \mathrm{m}$. It can be seen from figure 6(a) that during the first $2 \mathrm{~ns}$, the diameter of the streamer channel remains the same when the experimental condition changing from $-25 \mathrm{kV}, 6$ bar to $-35 \mathrm{kV}, 7$ bar. Start from $t=2.7 \mathrm{~ns}$, there were the protrusions appearing at $-35 \mathrm{kV}, 7$ bar. In this case, the diameter of the discharge channel shrunk immediately within $0.3 \mathrm{~ns}$ from $550 \mu \mathrm{m}$ to $50 \mu \mathrm{m}$ and stayed at the same low value in the next $7 \mathrm{~ns}$. For the experiments at $-25 \mathrm{kV}, 6$ bar nitrogen, the diameter of the discharge channel decrease in a slower way compared with experiments at higher voltage amplitude.

At $t=2 \mathrm{~ns}$, the streamer propagated to $500 \mu \mathrm{m}$ to the HV electrode. The FWHM of discharge channel $(630 \mu \mathrm{m})$ for $-25 \mathrm{kV}, 6$ bar is slightly higher than the diameter of discharge channel $(420 \mu \mathrm{m})$ for $-35 \mathrm{kV}, 7$ bar. For the same reason as the red curve in figure 6 (a), the diameter of the discharge channel shrunk rapidly due to the presence of protrusion. Both red and black curves went to the same diameter $(50 \mu \mathrm{m})$ in the end.

At the position of $1 \mathrm{~mm}$ to the $\mathrm{HV}$ electrode, at higher voltage $(-35 \mathrm{kV})$, the emission intensity of streamer propagation is not visible comparing with the emission from protrusions. In this case, the red curve in figure 6(c) starts at a low value which is close to the final diameter. While for the black curve, streamer zone still expand gradually at lower voltage and lower pressure conditions.

\section{Conclusion}

It is shown experimentally that, for both polarities, the initial stage of the discharge mainly gives the emission of the molecular bands. In a few nanoseconds later, the emission can transfer to the cw emission which mainly comes from the stepwise ionization and dissociation process. The diameter of the discharge propagation shrunk to approximate $50 \mu \mathrm{m}$ at the end of the streamer-to-filament transition process. 


\section{Acknowledgments}

The work was partially supported by LabEx Plas@Par, French National Research Agency (ASPEN Project) and the French-Russian international laboratory LIA KaPPA "Kinetics and physics of pulsed plasmas and their afterglow" (RFBR project 17-52-16001 and CNRS financial and organization support). The support of China Scholarship Council (CSC) for Chenyang Ding is gratefully acknowledged. The authors are thankful to Ali Mahjoub and Bruno Dufour for engineering support.

\section{References}

[1] Starikovskaia, S., Allegraud, K., Guaitella, O., Kosarev, I., Mintusov, E., Pendleton, S. J., Popov, N., Sagulenko, P., and Rousseau, A., "Surface discharges: possible applications for plasma-assisted ignition and electric field measurements," $48 t h$ AIAA Aerospace Sciences Meeting Including the New Horizons Forum and Aerospace Exposition, 2010, p. 1587.

[2] Kosarev, I., Khorunzhenko, V., Mintoussov, E., Sagulenko, P., Popov, N., and Starikovskaia, S., "A nanosecond surface dielectric barrier discharge at elevated pressures: time-resolved electric field and efficiency of initiation of combustion," Plasma Sources Science and Technology, Vol. 21, No. 4, 2012, p. 045012.

[3] Starikovskaia, S., "Plasma-assisted ignition and combustion: nanosecond discharges and development of kinetic mechanisms," Journal of Physics D: Applied Physics, Vol. 47, No. 35, 2014, p. 353001.

[4] Boumehdi, M. A., Stepanyan, S. A., Desgroux, P., Vanhove, G., and Starikovskaia, S. M., "Ignition of methane-and n-butanecontaining mixtures at high pressures by pulsed nanosecond discharge," Combustion and Flame, Vol. 162, No. 4, 2015, pp. $1336-1349$.

[5] Shcherbanev, S. A., Popov, N. A., and Starikovskaia, S. M., "Ignition of high pressure lean $\mathrm{H}_{2}$ : air mixtures along the multiple channels of nanosecond surface discharge," Combustion and Flame, Vol. 176, 2017, pp. 272-284.

[6] Ding, C., Khomenko, A. Y., Shcherbanev, S., and Starikovskaia, S., "Filamentary nanosecond surface dielectric barrier discharge. Experimental comparison of the streamer-to-filament transition for positive and negative polarities," Plasma Sources Science and Technology, Vol. 28, No. 8, 2019, p. 085005.

[7] Shcherbanev, S., Ding, C., Starikovskaia, S., and Popov, N., "Filamentary nanosecond surface dielectric barrier discharge. Plasma properties in the filaments," Plasma Sources Science and Technology, Vol. 28, No. 6, 2019, p. 065013.

[8] Shcherbanev, S., Khomenko, A. Y., Stepanyan, S., Popov, N., and Starikovskaia, S., "Optical emission spectrum of filamentary nanosecond surface dielectric barrier discharge,” Plasma Sources Science and Technology, Vol. 26, No. 2, 2017 , p. 02 LT01.

[9] Ding, C., Jean, A., Shcherbanev, S., Selivonin, I., Moralev, I., Popov, N. A., and Starikovskaia, S., "Experimental study of energy delivered to the filaments in high pressure nanosecond surface discharge," AIAA Scitech 2020 Forum, 2020 , p. 1662.

[10] Schindelin, J., Arganda-Carreras, I., Frise, E., Kaynig, V., Longair, M., Pietzsch, T., Preibisch, S., Rueden, C., Saalfeld, S., Schmid, B., et al., "Fiji: an open-source platform for biological-image analysis," Nature methods, Vol. 9, No. 7, 2012, pp. 676-682. 